two groups $(P>0.05)$. Further stratification analyses reveal that a significantly increased risk of colorectal neoplasia associated with hyperplastic polyps was evident among men $(\mathrm{OR}=$ $3.03,95 \% \mathrm{CI}=1.39-6.61)$ and subjects with $\mathrm{H}$. pylori infection $(\mathrm{OR}=1.88,95 \% \mathrm{CI}=1.01-3.54)$, compared with the control group.

Conclusions The risk of colorectal neoplasia increases in patients with sporadic gastric hyperplastic polyps, especially in male and H. pylori infection patients, and a screening colonoscopy is necessary for these patients to detect colorectal neoplasia.

\section{IDDF2019-ABS-0065 A SYSTEMATIC REVIEW IDENTIFIES INADEQUACIES IN THE REPORTING OF CROSSOVER TRIALS IN PROTON PUMP INHIBITORS CROSSOVER TRIALS}

${ }^{1}$ Qian Zhou*, ${ }^{2}$ Zhihang Chen, ${ }^{3}$ Jinxin Zhang, ${ }^{4}$ Sui Peng. 'Department of Medical Statistics, Clinical Trials Unit, The First Affiliated Hospital, Sun Yat-sen University, Guangzhou, China; ${ }^{2}$ Department of Liver Surgery, The First Affiliated Hospital, Sun Yat-sen University, Guangzhou, China; ${ }^{3}$ Department of Medical Statistics and Epidemiology, School of Public Health, Sun Yat-sen University, Guangzhou, China; ${ }^{4}$ Department of Gastroenterology, The First Affiliated Hospital, Sun Yat-sen University, Guangzhou, China

\subsection{6/gutjnl-2019-IDDFabstracts. 135}

Background Proton pump inhibitors (PPI) were widely used in acid-related diseases. Crossover design was frequently used in clinical trials of PPIs for its efficiency. Few studies were found to assess the quality of reporting of crossover trials for PPIs. Therefore, we aimed to review crossover trials for PPIs and evaluated the quality of reporting of these crossover trials.

Methods We searched Pubmed for all crossover trials related to PPIs published between January 1999 and June 2018 and evaluated the quality of reporting. Furthermore, two scores with the same range which have been published to evaluating the risk of bias were applied and the relationship and agreement between the two scores were analyzed.

Results In total, 74 articles which met our inclusion criteria were included, consisting of 2336 study participants in total. Most of the trials (71.6\%) had a washout period of larger or equal than eight days, but only 8 of them making justification. Only 14 of 74 studies evaluated the carry-over effect. In more than 50\% articles, sample size calculation has not been mentioned. There was a significant difference in sample size between trials with and without sample size estimation (36 $(24,45)$ vs $21(12,30), \mathrm{p}<0.001)$. Paired data test $(40.6 \%)$ and analysis of variance with random effect or mixed model $(40.5 \%)$ were the most two frequently used statistical methods for primary analysis. Median Gewandter score was higher than the median Ding score $(8(6,9)$ vs $7(5,8)$, Wilcoxon signed-rank test, $\mathrm{p}<0.001)$. Although the Spearman coefficient between the scores were $0.72(\mathrm{p}<0.001)$, the Kappa coefficient was low with 0.357 (95\% CI: 0.196-0.519, McNemar test, $\mathrm{P}<0.001$ ) based on groups using both seven as a cutoff. In general, no significant improvement found over time in most evaluating aspects. A recommendation table for reporting crossover trials in PPIs was given according to the deficiencies from the results.

Conclusions These findings demonstrated general deficiencies in crossover trials for PPI. In particular, evaluation of carryover effects should be enhanced. Currently, no strong consensus was reached on reporting of crossover trials, requiring guidelines for proper reporting of crossover trials.

\section{IDDF2019-ABS-0071 ANALYSIS OF THE EFFECT OF SUBMUCOSAL INJECTION ON ENDOSCOPIC RESECTION OF AMPULLARY ADENOMA}

Wenhai Wang*, Peng Li, Yongjun Wang, Fujing Lv, Li Yu, Yinglin Niu, Wei Li, Shengtao Zhu, Ming Ji. Department of Gastroenterology, Beijing Friendship Hospital, Capital Medical University, China

\subsection{6/gutjnl-2019-IDDFabstracts.136}

Background Endoscopic papillectomy(EP) is currently well accepted for treating ampullary adenoma alternating surgery. Submucosal injection should be performed or not before EP is still uncertain because of the structure and special location of duodenal papilla.

Objective To investigate and analyze the effect of EP on submucosal injection of ampullary adenoma.

Methods 43 patients who underwent EP at Digestive Endoscopy Center of Beijing Friendship Hospital from September 2012 to January 2018 were retrospectively recruited, including 23 males and 20 females, aged from 28 to 79 years. The patients were subdivided into two groups, 22 patients in the submucosal injection group including 12 males and 10 females, and 21 patients in the control group including 11 males and 10 females. Patient demographics, baseline blood test, tumor characteristics, en bloc resection rate, complete resection rate, complication rate and other endoscopic treatment data were collected. The primary endpoint was postprocedure pancreatitis.

Results There were no significant differences in gender $\left(\chi^{2}\right.$ $=0.903, \mathrm{P}=0.342)$, age $(\mathrm{t}=0.142, \mathrm{P}=0.383)$ and tumor size $(t=0.938, P=0.435)$ between two groups. En bloc resection rate and complete resection rate were $91.67 \%$ and $95.83 \%$ respectively in the submucosal injection group, and were $80 \%$ and $100 \%$ in the control group. There were no significant differences with regard to en bloc resection rate $\left(\chi^{2}=1.124\right.$, $\mathrm{P}=0.289)$ and complete excision rate $\left(\chi^{2}=0.641, \mathrm{P}=0.423\right)$ between two groups. No perforation or severe postoperative pancreatitis occurred in all the patients. Intraoperative bleeding occurred in 3 cases $(13.6 \%)$ of the submucosal injection group and 3 cases $(14.3 \%)$ of the control group, and 2 cases of hyperamylasemia in the submucosal injection group and none in the control group. No significant differences exist between the two groups according to intraoperative bleeding $\left(\chi^{2}=0.006\right.$, $\mathrm{P}=0.940)$ and hyperamylasemia $\left(\chi^{2}=1.318, \mathrm{P}=0.251\right)$.

Conclusions Our data suggest that routine submucosal injection in patients undergoing endoscopic papillectomy may not be necessary and large-scale prospective multicenter randomized studies are required to identify the subgroup of patients who would benefit.

\section{IDDF2019-ABS-0075 SERUM METABONOMIC ANALYSIS AND ESTABLISHMENT OF DIAGNOSTIC MODEL OF PANCREATIC CANCER ASSOCIATED DIABETES}

Xiangyi He*, Yaozong Yuan. Department of Gastroenterology, Ruijin Hospital, Shanghai Jiao Tong University School of Medicine, China

\subsection{6/gutjnl-2019-IDDFabstracts. 137}

Background For the lack of effective screening tool on newonset diabetic mellitus for pancreatic cancer (PC), the current study aimed to establish a diagnostic model based on serum 
Abstract IDDF2019-ABS-0075 Table 1 Differentially expressed metabolites between $\mathrm{pc}$ and $\mathrm{dm}$

\begin{tabular}{llllll}
\hline name & ttest & $\begin{array}{l}\text { fold } \\
\text { Change } \\
\text { (PC/DM) }\end{array}$ & VIP & mass & Mode \\
& & & & \\
\hline C16 & 0.000 & 0.57 & 2.192 & 351.2167 & Neg \\
Sphingosine-1-phosphate & & & & & \\
keto palmitic acid & 0.000 & 6.15 & 2.536 & 270.2185 & Neg \\
Isoleucine & 0.000 & 0.76 & 2.260 & 131.0945 & Neg \\
N-Succinyl-L- & 0.000 & 0.19 & 2.880 & 290.1123 & Neg \\
diaminopimelic acid & & & & & \\
Uridine & 0.000 & 0.67 & 2.056 & 244.0692 & Neg \\
3-ketosphingosine & 0.000 & 4.10 & 2.500 & 297.2674 & Pos \\
Arachidonoyl dopamine & 0.000 & 0.48 & 2.319 & 439.3074 & Pos \\
PE(18:2) & 0.000 & 0.58 & 2.594 & 477.2868 & Pos \\
Ubiquinone-1 & 0.000 & 0.38 & 2.939 & 250.121 & Pos \\
Valine & 0.007 & 0.81 & 2.573 & 117.0792 & Pos \\
\hline
\end{tabular}

metabolomics to discriminate pancreatic cancer (PC) from new onset diabetic mellitus.

Methods Serum samples from patients with new-onset diabetic mellitus ( $\mathrm{n}=30)$ and new onset diabetic mellitus with PC $(\mathrm{N}=30)$ were examined by liquid chromatography-mass spectrometry (LC-MS). Data were analyzed using orthogonal projection to latent structures (OPLS) and t-test for the differentially expressed metabolites between two groups. The diagnostic model was established using Logistic regression via a stepwise method on the first 15 new onset diabetic mellitus and new onset diabetic mellitus with PC training set. It was then evaluated in the late 15 new onset diabetic mellitus and new onset diabetic mellitus with PC testing set.

Results Five metabolites identified in positive ion model were 3-ketosphingosine, Arachidonoyl dopamine, PE(18:2), Ubiquinone-1 and Valine. C16 Sphingosine-1-phosphate, keto palmitic acid, Isoleucine, N-Succinyl-L-diaminopimelic acid and Uridine in negative ion model were identified to be differentially expressed in the two groups. The regression model established in training set was $\mathrm{p}=\mathrm{e}(\mathrm{X} \beta) /(1+\mathrm{e}(\mathrm{X} \beta)),(\mathrm{X} \beta)=$ $158.975-1.891$ (Age) +0.309 (PE18:2) + 1.035 (C16_Sphingosine_1_phosphate) + 0.084 (Isoleucine) + 1.1145 ( N_Succinyl_L_diaminopimelic_acid). The AUC of this model was 0.982 in testing set; the sensitivity and specificity were both $93.3 \%$. (table 1).

Conclusions Serum metabolomics-based diagnostic approach is a promising method for screening PC from new onset diabetic mellitus.

\section{IDDF2019-ABS-0083 THE USE OF HEMOSPRAY ENDOTHERAPY IN PATIENTS WITH ACUTE GASTROINTESTINAL BLEEDING: A PHILIPPINE EXPERIENCE}

Enrik John Aguila*, Juliet Cervantes, Joseph Erwin Dumagpi. St. Luke's Medical Center Global City, Philippines

\subsection{6/gutjnl-2019-IDDFabstracts. 138}

Background Hemospray (TC-325) is a novel hemostatic agent recently licensed for endoscopic hemostasis of non-variceal upper gastrointestinal (GI) bleeding in Europe, Canada and America. It acts by creating a mechanical barrier and effecting hemostasis on actively bleeding lesions via endoscopy. After an extensive search of publications, the group only found one research describing the experience of hemospray use in the local Asian setting done in 2011. This study aims to describe the effectiveness of hemospray therapy on patients treated at the St. Luke's Medical Center, Global City (SLMC GC), Philippines.

Methods A retrospective study was done on consecutive adults with upper and lower active gastrointestinal tract bleeding treated with hemospray at SLMC GC since its introduction in 2017. Data on patient profile, details of the procedure, and indications of hemospray use either as monotherapy or as salvage therapy were collected. Outcomes including primary hemostasis and re-bleeding rates were analyzed.

Results From 2017 to 2018, a total of 8 patients with acute GI bleeding were managed with hemospray at SLMC GC. With an age range of 36-83 years old, these patients presented with hematochezia, hematemesis or melena. $\underline{3}$ of the cases had a bleeding gastric mass, 3 had bleeding ulcers, while 2 had portal hypertensive bleeding. Hemospray powder was applied on the identified bleeding sites via endoscopy (figure 1A)(figure 1B.). The rate of successful initial hemostasis after hemospray was $87.5 \%$ (7/8). In terms of the endoscopic hemostatic methods used, of the 8 patients, 4 (50\%) had
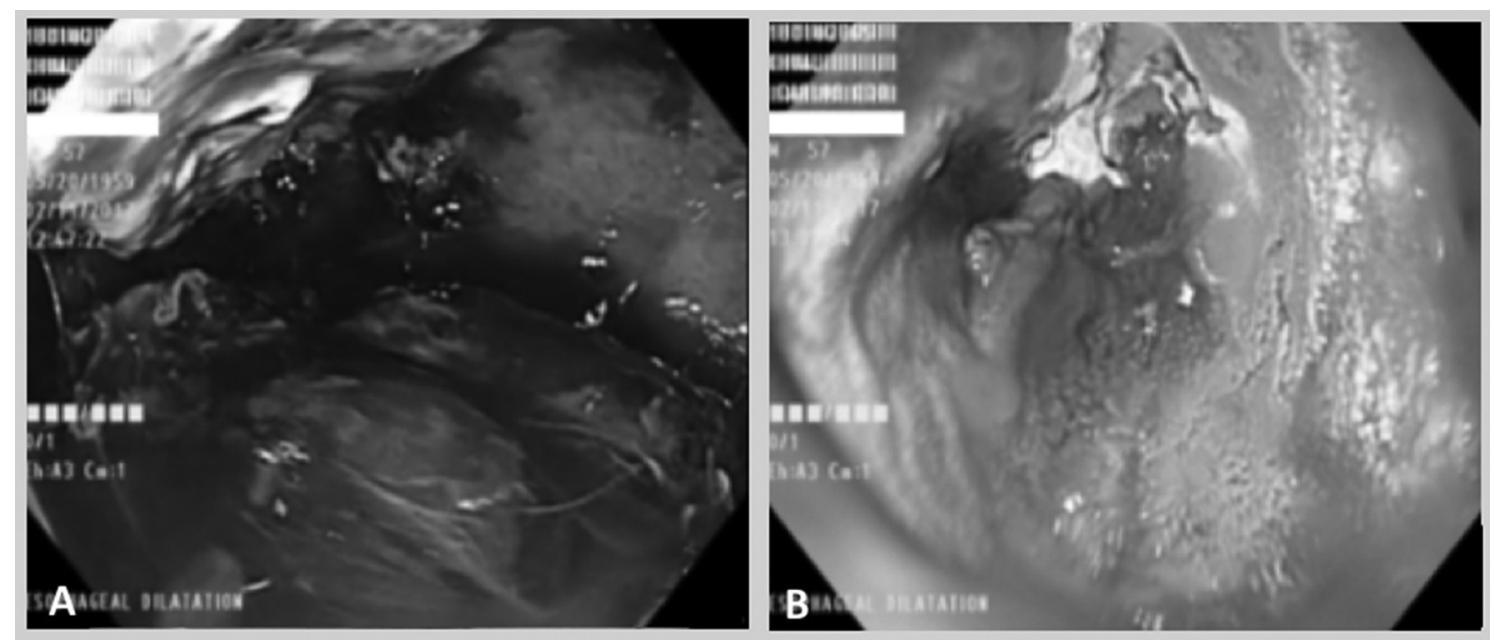

Abstract IDDF2019-ABS-0083 Figure 1 A) Ulcerating gastric mass at the corpus; B) Successful hemostasis after hemospray application 